5-1-2016

\title{
Liu-Type Logistic Estimators with Optimal Shrinkage Parameter
}

Yasin Asar

Necmettin Erbakan University, yasar@konya.edu.tr

Follow this and additional works at: http://digitalcommons.wayne.edu/jmasm

Part of the Applied Statistics Commons, Social and Behavioral Sciences Commons, and the Statistical Theory Commons

\section{Recommended Citation}

Asar, Yasin (2016) "Liu-Type Logistic Estimators with Optimal Shrinkage Parameter," Journal of Modern Applied Statistical Methods: Vol. 15 : Iss. 1 , Article 36.

DOI: $10.22237 /$ jmasm/1462077300

Available at: http://digitalcommons.wayne.edu/jmasm/vol15/iss1/36

This Regular Article is brought to you for free and open access by the Open Access Journals at DigitalCommons@WayneState. It has been accepted for inclusion in Journal of Modern Applied Statistical Methods by an authorized editor of DigitalCommons@WayneState. 


\title{
Liu-Type Logistic Estimators with Optimal Shrinkage Parameter
}

\author{
Yasin Asar \\ Necmettin Erbakan University \\ Konya, Turkey
}

\begin{abstract}
Multicollinearity in logistic regression affects the variance of the maximum likelihood estimator negatively. In this study, Liu-type estimators are used to reduce the variance and overcome the multicollinearity by applying some existing ridge regression estimators to the case of logistic regression model. A Monte Carlo simulation is given to evaluate the performances of these estimators when the optimal shrinkage parameter is used in the Liutype estimators, along with an application of real case data.
\end{abstract}

Keywords: Logistic regression, multicollinearity, maximum likelihood, MSE, Liutype estimator

\section{Introduction}

It is a very common problem to deal with highly intercorrelated explanatory variables. Especially in economics and in other applied research areas, the variables used in the multiple linear regression models are collinear. This problem is called multicollinearity. There are some results of the multicollinearity problem such as having inflated variance scores and instable estimations of the parameters when the ordinary least square (OLS) estimator is used. Similarly, in the logistic regression model, if the maximum likelihood method is used, these drawbacks occur at all. Also, one cannot obtain decisive answers to the related questions in both of the models.

There are some methods to deal with this problem. One method is to use ridge regression, first introduced by Hoerl and Kennard (1970). The other methods are to use Liu or Liu-type estimators proposed by Liu (1993) and Liu (2003) respectively.

These methods have been applied in the case of multiple linear models. However, there is not much attention paid to the multicollinearity problem in the case of logistic regression. Månsson and Shukur (2011), Kibria, Månsson, and

Mr. Asar is an Assistant Professor in the Department of Mathematics-Computer Science. Email himat: yasar@konya.edu.tror at yasinasar@hotmail.com. 


\section{YASIN ASAR}

Shukur (2012), and Inan and Erdogan (2013) are some exceptions. In the first two studies, the authors used some early defined ridge estimators in the logistic regression model. In the last one, the authors applied Liu-type estimators given in Liu (1993) to the logistic model as well.

There is another Liu-type estimator defined by Huang (2012) for the logistic regression model. The author explained the theoretical advantages of this estimator and gave some comparisons. In this study, we use this estimator with optimal shrinkage parameter and some existing ridge parameters in order to make a simulation study to see the performance of these estimators in the logistic regression model.

\section{Methodology}

Consider the binary logistic regression model, a widely used method in statistical analysis, such that the dependent variable is $\operatorname{Be}(\mathbf{P})$ where $\mathbf{P}=\mathrm{e}^{\mathbf{x} \boldsymbol{\beta}} / 1+\mathrm{e}^{\mathbf{x} \boldsymbol{\beta}}$ such that $\mathbf{X}$ is the design matrix of order $n \times(p+1), p$ is the number of explanatory variables, and $\boldsymbol{\beta}$ is the coefficient vector of order $(p+1) \times 1$. The most commonly applied method of estimating $\boldsymbol{\beta}$ is the maximum likelihood estimation (MLE) method.

One can compute the MLE of $\boldsymbol{\beta}$ by using the iteratively re-weighted least square algorithm as follows:

$$
\hat{\boldsymbol{\beta}}_{\mathrm{MLE}}=\left(\mathbf{X}^{\prime} \mathbf{W} \mathbf{X}\right)^{-1} \mathbf{X}^{\prime} \mathbf{W} \hat{\mathbf{z}}
$$

where $\mathbf{W}=\operatorname{diag}\left(P_{i}\left(1-P_{i}\right)\right)$ and $\hat{z}_{i}=\log \left(P_{i}\right)+y_{i}-P_{i} / P_{i}\left(1-P_{i}\right)$ is the $i^{\text {th }}$ element of the vector $\hat{\mathbf{z}}, i=1,2, \ldots, n$.

The mean square error (MSE) of the MLE is given as follows:

$$
\operatorname{MSE}\left(\hat{\boldsymbol{\beta}}_{\mathrm{MLE}}\right)=\mathrm{E}\left[\left(\hat{\boldsymbol{\beta}}_{\mathrm{MLE}}-\boldsymbol{\beta}\right)^{\prime}\left(\hat{\boldsymbol{\beta}}_{\mathrm{MLE}}-\boldsymbol{\beta}\right)\right]=\operatorname{tr}\left(\mathbf{X}^{\prime} \mathbf{W X}\right)^{-1}=\sum_{j=1}^{p+1} \frac{1}{\lambda_{j}},
$$

where $\lambda_{j}$ is the $j^{\text {th }}$ eigenvalue of the matrix $\mathbf{X}^{\prime} \mathbf{W X}$.

If some of the eigenvalues are small (close to zero), then the asymptotic variance of MLE becomes inflated. In other words, multicollinearity between the 


\section{LIU-TYPE LOGISTIC ESTIMATORS}

explanatory variable makes this estimator instable. Schaefer, Roi, and Wolfe (1984) proposed the following logistic ridge estimators to cure this problem:

$$
\hat{\boldsymbol{\beta}}_{k}=\left(\mathbf{X}^{\prime} \mathbf{W X}+k \mathbf{I}\right)^{-1} \mathbf{X}^{\prime} \mathbf{W} \mathbf{X} \hat{\boldsymbol{\beta}}_{\mathrm{MLE}},
$$

where

$$
k=\frac{1}{\hat{\boldsymbol{\beta}}_{\mathrm{MLE}}^{\prime} \hat{\boldsymbol{\beta}}_{\mathrm{MLE}}}, \frac{p}{\hat{\boldsymbol{\beta}}_{\mathrm{MLE}}^{\prime} \hat{\boldsymbol{\beta}}_{\mathrm{MLE}}}, \frac{p+1}{\hat{\boldsymbol{\beta}}_{\mathrm{MLE}}^{\prime} \hat{\boldsymbol{\beta}}_{\mathrm{MLE}}}
$$

The following logistic Liu estimator $\hat{\boldsymbol{\beta}}_{d}$ was defined in Månsson, Kibria, and Shukur (2012):

$$
\hat{\boldsymbol{\beta}}_{d}=\left(\mathbf{X}^{\prime} \mathbf{W X}+\mathbf{I}\right)^{-1}\left(\mathbf{X}^{\prime} \mathbf{W} \mathbf{X}+d \mathbf{I}\right) \hat{\boldsymbol{\beta}}_{\mathrm{MLE}},
$$

where $0<d<1$. Also, Huang (2012) defined the estimator $\hat{\boldsymbol{\beta}}(k, d)$ as a combination of the two different estimators given above such that

$$
\hat{\boldsymbol{\beta}}(k, d)=\left(\mathbf{X}^{\prime} \mathbf{W} \mathbf{X}+k \mathbf{I}\right)^{-1}\left(\mathbf{X}^{\prime} \mathbf{W} \mathbf{X}+k d \mathbf{I}\right) \hat{\boldsymbol{\beta}}_{\mathrm{MLE}},
$$

where $k>0,0<d<1$. It was shown that: if $d=1$, then $\hat{\boldsymbol{\beta}}(k, d)=\hat{\boldsymbol{\beta}}_{\mathrm{MLE}}$; if $k=0$, then $\hat{\boldsymbol{\beta}}(k, d)=\hat{\boldsymbol{\beta}}_{\mathrm{MLE}}$; and if $k=1, \hat{\boldsymbol{\beta}}(k, d)=\hat{\boldsymbol{\beta}}_{d}$. In Månsson et al. (2012), it was proved that, when $d<1,\left\|\hat{\boldsymbol{\beta}}_{d}\right\|<\left\|\hat{\boldsymbol{\beta}}_{\mathrm{MLE}}\right\|$; it was also shown that $\hat{\boldsymbol{\beta}}_{d}$ has a better performance than $\hat{\boldsymbol{\beta}}_{\mathrm{MLE}}$ in the presence of multicollinearity.

In order to provide the explicit form of the MSE function of $\hat{\boldsymbol{\beta}}(k, d)$ use the following transformations:

Let $\quad \boldsymbol{\alpha}=\mathbf{Q}^{\prime} \boldsymbol{\beta}, \quad \mathbf{Q}^{\prime} \mathbf{X}^{\prime} \mathbf{W X} \mathbf{Q}=\boldsymbol{\Lambda}=\operatorname{diag}\left(\lambda_{1}, \lambda_{2}, \ldots, \lambda_{p+1}\right), \quad$ where $\lambda_{1} \geq \lambda_{2} \geq \cdots \geq \lambda_{p+1}>0$ such that the $\lambda_{j}$ 's are the eigenvalues of the matrix $\mathbf{X}^{\prime} \mathbf{W X}$ and $\mathbf{Q}$ is the matrix whose columns are the eigenvectors of the matrix $\mathbf{X}^{\prime} \mathbf{W X}$. $\left(\mathbf{X}^{\prime} \mathbf{W X}=\mathbf{Q}^{\prime} \mathbf{\Lambda} \mathbf{Q}\right)$.

The MSE function of $\hat{\boldsymbol{\beta}}(k, d)$ is as follows: 


\section{YASIN ASAR}

$$
\begin{aligned}
\operatorname{MSE}(\hat{\boldsymbol{\beta}}(k, d)) & =\sum_{j=1}^{p+1} \frac{\left(\lambda_{j}+k d\right)^{2}}{\lambda_{j}\left(\lambda_{j}+k\right)^{2}}+\sum_{j=1}^{p+1} \frac{k^{2}(1-d)^{2} \alpha_{j}^{2}}{\left(\lambda_{j}+k\right)^{2}} \\
& =\mathrm{f}_{1}(k, d)+\mathrm{f}_{2}(k, d)
\end{aligned}
$$

where $\mathrm{f}_{1}(k, d)$ is the variance function and $\mathrm{f}_{2}(k, d)$ is the squared bias. Thus choose suitable values for the parameters $k$ and $d$ in order to obtain a less MSE value than that of MLE.

Three theorems given in Huang (2012) are presented about the properties of the estimator $\hat{\boldsymbol{\beta}}(k, d)$ :

Theorem 1. The asymptotic variance $\mathrm{f}_{1}(k, d)$ and the squared bias $\mathrm{f}_{2}(k, d)$ are two continuous functions of $k$ and $d$; for fixed $d^{*}, 0<d^{*}<1, \mathrm{f}_{1}\left(k, d^{*}\right)$ and $\mathrm{f}_{2}\left(k, d^{*}\right)$ are monotonically decreasing and increasing functions of $k$, respectively; for a fixed $k^{*}>0, \mathrm{f}_{1}\left(k^{*}, d\right)$ and $\mathrm{f}_{2}\left(k^{*}, d\right)$ are monotonically increasing and decreasing functions of $k$, respectively (Huang, 2012).

Theorem 2. For a fixed $d^{*}, 0<d_{*}<1$, there exists a $k>0$ such that $\operatorname{MSE}(\hat{\boldsymbol{\beta}}(k, d))<\operatorname{MSE}\left(\hat{\boldsymbol{\beta}}_{\text {MLE }}\right)($ Huang, 2012).

Theorem 3. If $k>0$ and $0<d<1$, then $\operatorname{MSEM}(\hat{\boldsymbol{\beta}}(k, d))<\operatorname{MSEM}\left(\hat{\boldsymbol{\beta}}_{\mathrm{MLE}}\right)$ if and only if $k(1-d) \boldsymbol{\alpha}^{\prime}\left[k(1+d) \boldsymbol{\Lambda}^{-1}+2 \mathbf{I}\right]^{-1} \boldsymbol{\alpha}<1$, where $\operatorname{MSEM}(\tilde{\boldsymbol{\beta}})=\mathrm{E}\left[(\tilde{\boldsymbol{\beta}}-\boldsymbol{\beta})(\tilde{\boldsymbol{\beta}}-\boldsymbol{\beta})^{\prime}\right]$, $\tilde{\boldsymbol{\beta}}=\hat{\boldsymbol{\beta}}_{\mathrm{MLE}}, \hat{\boldsymbol{\beta}}(k, d)$.

These theorems show the theoretical advantage of the estimator $\hat{\boldsymbol{\beta}}(k, d)$. In Huang (2012), the author designed a simulation study considering $k=0.1,0.3$, and 0.5 and $d=0.2,0.4$, and 0.6. However, it is anticipated that optimal value of $d$ and some estimators of $k$ will result in better performance.

Note that the values of the parameter $d$ are restricted to the interval $0<d<1$ in the definition of $\hat{\boldsymbol{\beta}}(k, d)$ in order to obtain a sufficient condition satisfying $\operatorname{MSE}(\hat{\boldsymbol{\beta}}(k, d))<\operatorname{MSE}\left(\hat{\boldsymbol{\beta}}_{\mathrm{MLE}}\right)$. However, in the simulation given in Huang (2012), the author states that MSE of $\hat{\boldsymbol{\beta}}(k, d)$ can be smaller than MSE of $\hat{\boldsymbol{\beta}}_{\mathrm{MLE}}$ without satisfying this sufficient condition. Thus, we expand the restriction on $d$ and 


\section{LIU-TYPE LOGISTIC ESTIMATORS}

conduct our simulation study such that the optimal parameter $d_{\mathrm{opt}}$ satisfies the following conditions: $-\infty<d_{\mathrm{opt}}<\infty, d_{\mathrm{opt}} \neq 0$, and $d_{\mathrm{opt}} \neq 1$. In the following theorem, $d_{\text {opt }}$ is presented.

Theorem 4. The optimal shrinkage parameter $-\infty<d_{\mathrm{opt}}<\infty$ for minimizing $\operatorname{MSE}(\hat{\boldsymbol{\beta}}(k, d)) \forall k>0$ is the following:

$$
d_{\mathrm{opt}}=\frac{\sum_{j=1}^{p+1} \frac{k \alpha_{j}^{2}-1}{\left(\lambda_{j}+k\right)^{2}}}{\sum_{j=1}^{p+1} \frac{k\left(1+\lambda_{j} \alpha_{j}^{2}\right)}{\lambda_{j}\left(\lambda_{j}+k\right)^{2}}}
$$

Proof: It is easy to find the optimal parameter $d_{\mathrm{opt}}$ by differentiating $\operatorname{MSE}(\hat{\boldsymbol{\beta}}(k, d))$ with respect to $d$ and equating the derivative to zero. Solving the equation for $d$, we get the optimal parameter $d_{\text {opt }}$.

After choosing the optimal parameter $d_{\text {opt }}$ for $d$, the parameter $k$ must be selected. In literature, there are some estimators for the selection of $k$. The followings are the estimators of $k$ that are used in the simulation study:

1. $\quad k_{1}=\frac{p+1}{\hat{\boldsymbol{\beta}}_{\mathrm{MLE}}^{\prime} \hat{\boldsymbol{\beta}}_{\mathrm{MLE}}}($ Schaefer et al., 1984)

2. $\quad k_{2}=\frac{p}{\hat{\boldsymbol{\beta}}_{\mathrm{MLE}}^{\prime} \hat{\boldsymbol{\beta}}_{\mathrm{MLE}}}($ Schaefer et al., 1984)

3. $k_{3}=\frac{\hat{\sigma}^{2}}{\hat{\alpha}_{\max }^{2}}$, where $\hat{\alpha}_{\max }$ is the maximum element of $\hat{\boldsymbol{\alpha}}_{\mathrm{MLE}}=\mathbf{Q}^{\prime} \hat{\boldsymbol{\beta}}_{\mathrm{MLE}}$ and $\hat{\sigma}^{2}=(\mathbf{y}-\hat{\mathbf{y}})^{\prime}(\mathbf{y}-\hat{\mathbf{y}}) / n-p-1($ Hoerl \& Kennard, 1970)

4. $\quad k_{4}=\frac{1}{\hat{\alpha}_{\max }^{2}}$, which is a modified version of $k_{3}$ (Schaefer et al., 1984)

5. $\quad k_{5}=\frac{\hat{\sigma}^{2}}{\left(\prod_{j=1}^{p+1} \hat{\alpha}_{j}^{2}\right)^{\frac{1}{p+1}}}$, which is the geometric mean of $k_{j}=\hat{\sigma}^{2} / \hat{\alpha}_{j}^{2}$

(Kibria, 2003) 


\section{YASIN ASAR}

6. $\quad k_{6}=\operatorname{median}\left(\frac{\hat{\sigma}^{2}}{\hat{\alpha}_{j}^{2}}\right)($ Kibria, 2003)

7. $k_{7}=\max \left(\frac{\lambda_{j} \hat{\sigma}^{2}}{(n-p-1) \hat{\sigma}^{2}+\lambda_{j} \hat{\alpha}_{j}^{2}}\right)$ (Alkhamisi, Khalaf, \& Shukur, 2006)

8. $\quad k_{8}=\max \left(\frac{1}{\sqrt{\hat{\sigma}^{2} / \hat{\alpha}_{j}^{2}}}\right)($ Muniz \& Kibria, 2009)

Because the $\alpha_{j}^{2}$ 's and $\sigma^{2}$ are not known in practice, the estimators $\hat{\alpha}_{j}^{2}$ and $\hat{\sigma}^{2}$ are used in the above formulae.

\section{Monte Carlo Simulation}

The effective factors are chosen to be the number of explanatory variables $p$, the sample size $n$, and the correlation among the explanatory variables $\rho^{2}$. MSE and mean absolute error (MAE) are used as the criterion of judgment.

The average MSE and MAE of the estimators $\hat{\boldsymbol{\beta}}_{\mathrm{MLE}}$ and $\hat{\boldsymbol{\beta}}\left(k, d_{\mathrm{opt}}\right)$ for $k=k_{1}, \ldots, k_{8}$ are computed by using the following equations:

$$
\begin{aligned}
\operatorname{AMSE}(\hat{\boldsymbol{\beta}}) & =\sum_{r=1}^{3000} \frac{\left(\hat{\boldsymbol{\beta}}_{r}-\boldsymbol{\beta}\right)^{\prime}\left(\hat{\boldsymbol{\beta}}_{r}-\boldsymbol{\beta}\right)}{3000} \\
\operatorname{MAE}\left(\hat{\boldsymbol{\beta}}_{r}\right) & =\sum_{r=1}^{3000} \frac{\left|\hat{\boldsymbol{\beta}}_{r}-\boldsymbol{\beta}\right|}{3000},
\end{aligned}
$$

where $\hat{\boldsymbol{\beta}}_{r}=\hat{\boldsymbol{\beta}}_{\mathrm{MLE}}, \hat{\boldsymbol{\beta}}\left(k, d_{\mathrm{opt}}\right)$ at the $r^{\text {th }}$ step of the simulation.

Following Kibria (2003), in order to generate the explanatory variables, the following equation is used:

$$
x_{i j}=\left(1-\rho^{2}\right)^{\frac{1}{2}} z_{i j}+\rho z_{i p}
$$




\section{LIU-TYPE LOGISTIC ESTIMATORS}

where $i=1,2, \ldots, n$ and $j=1,2, \ldots, p+1$, and the $z_{i j}$ 's are pseudo-random numbers following the standard normal distribution.

The dependent variable is obtained by using $\operatorname{Be}(\mathbf{P})$, where $P_{i}=\mathrm{e}^{x_{i} \beta} / 1+\mathrm{e}^{x_{i} \beta}$ such that $x_{i}$ is the $i^{t \text { th }}$ row of the design matrix $\mathbf{X}$. The parameters $\boldsymbol{\beta}_{1}, \boldsymbol{\beta}_{2}, \ldots, \boldsymbol{\beta}_{p+1}$ are chosen due to Newhouse and Oman (1971) such that $\boldsymbol{\beta}^{\prime} \boldsymbol{\beta}=1$, which is a commonly used restriction in many simulation studies in the field; for example see Alkhamisi and Shukur (2008), Asar, Karaibrahimoğlu, and Genç (2014), and Kibria (2003).

The following cases are considered: $\rho^{2}=0.90,0.95$, and $0.99, n=50,100$, and 200, and $p=4,8$, and 12 . The simulation is repeated 3000 times for each set of $\left(\rho^{2}, n, p\right)$. Thus, via this set up, it may be determined which of the estimators $k_{1}, \ldots, k_{8}$ has better performance when $d_{\text {opt }}$ is used for different combinations of $\left(\rho^{2}, n, p\right)$.

\section{Results and Discussion}

Results of the Monte Carlo simulation are compiled in Tables 1-6. The factors affecting the MSE's of the estimators in the simulation are the degree of correlation $\rho$, the sample size $n$, and the number of explanatory variables $p$.

Tables 1, 3, and 5 are the AMSE values of Liu-type estimators, MLE for different values of $k$, and optimal shrinkage parameter $d_{\text {opt }}$ when $p=4,8$, and 12 . According to these tables, when $n$ and $p$ are fixed, the increase in the correlation $\rho$ causes an increase in the AMSE values of the estimators without exception. When $p=12$, the increase in the correlation inflates the AMSE values drastically. The worst case is obtained when the sample size is low and the degree of correlation is high, namely, $n=50$ and $\rho=0.99$. When the sample size $n$ is increased, fixing $p$ and $\rho$, it has a positive effect on estimators; in other words, it can be seen that the AMSE values decreases for all of the estimators. Especially for MLE, there is a rapid decrease in the case of high correlation. When fixing $n$ and $\rho$, if the number of explanatory variables is focused upon, it is observed that an increase in the value of $p$ corresponds to an increase in the AMSE values for both MLE and the other estimators. When the number of explanatory variables is increased, one should also increase the sample size sufficiently in order to make stable estimations. The estimator having best performance among others is $k_{8}$ for all of the situations.

In Tables 2, 4 and 6, the MAE values are presented for the estimators for different values of $k$ and optimal shrinkage parameter $d_{\text {opt }}$ when $p=4,8$, and 12 . Similar comments apply in the case of AMSE. However, the only difference is that the MAE values are significantly smaller than AMSE values for all of the cases. 


\section{YASIN ASAR}

Particularly for the case $p=12$, the MAE values are much smaller than the AMSE values. Again, $k_{8}$ is the best option if MAE is used as a performance criterion.

Table 1. The AMSE values for different $k$ with optimal $d$ for $p=4$

\begin{tabular}{|c|c|c|c|c|c|c|c|c|c|}
\hline \multirow{2}{*}{$\begin{array}{l}\rho \\
n\end{array}$} & \multicolumn{3}{|c|}{0.9} & \multicolumn{3}{|c|}{0.95} & \multicolumn{3}{|c|}{0.99} \\
\hline & 50 & 100 & 200 & 50 & 100 & 200 & 50 & 100 & 200 \\
\hline$k_{1}$ & 1.1453 & 0.5309 & 0.4556 & 2.4285 & 0.9978 & 0.6572 & 10.5534 & 3.0258 & 1.5615 \\
\hline$k_{2}$ & 1.1650 & 0.5440 & 0.4647 & 2.4853 & 1.0291 & 0.6742 & 10.7168 & 3.0939 & 1.5964 \\
\hline$k_{3}$ & 1.3392 & 0.6530 & 0.5187 & 3.0932 & 1.2907 & 0.7937 & 12.8152 & 4.1027 & 2.0750 \\
\hline$k_{4}$ & 1.2258 & 0.5760 & 0.4791 & 2.6704 & 1.1247 & 0.7122 & 11.3301 & 3.3589 & 1.7338 \\
\hline$k_{5}$ & 1.2328 & 0.5734 & 0.4712 & 2.7752 & 1.1296 & 0.7079 & 11.3754 & 3.4391 & 1.7882 \\
\hline$k_{6}$ & 1.2192 & 0.6070 & 0.4969 & 2.5722 & 1.1291 & 0.7425 & 10.1046 & 3.0120 & 1.6530 \\
\hline$k_{7}$ & 1.2911 & 0.6498 & 0.5196 & 2.8291 & 1.2536 & 0.7898 & 10.5026 & 3.3305 & 1.8326 \\
\hline$k_{8}$ & 1.0532 & 0.5352 & 0.4600 & 2.1244 & 0.8674 & 0.6469 & 9.4613 & 2.6145 & 1.3765 \\
\hline MLE & 3.1825 & 1.1528 & 0.8809 & 7.5693 & 2.6893 & 1.3873 & 31.3891 & 11.2257 & 5.3554 \\
\hline
\end{tabular}

Table 2. The MAE values for different $k$ with optimal $d$ for $p=4$

\begin{tabular}{|c|c|c|c|c|c|c|c|c|c|}
\hline \multirow{2}{*}{$\frac{\rho}{n}$} & \multicolumn{3}{|c|}{0.9} & \multicolumn{3}{|c|}{0.95} & \multicolumn{3}{|c|}{0.99} \\
\hline & 50 & 100 & 200 & 50 & 100 & 200 & 50 & 100 & 200 \\
\hline$k_{1}$ & 0.9224 & 0.7068 & 0.6605 & 1.1921 & 0.9009 & 0.7785 & 2.1122 & 1.3928 & 1.1036 \\
\hline$k_{2}$ & 0.9319 & 0.7155 & 0.6674 & 1.2168 & 0.9187 & 0.7895 & 2.1498 & 1.4144 & 1.1182 \\
\hline$k_{3}$ & 1.0211 & 0.7872 & 0.7069 & 1.4623 & 1.0578 & 0.8626 & 2.6491 & 1.7357 & 1.3122 \\
\hline$k_{4}$ & 0.9613 & 0.7367 & 0.6779 & 1.2913 & 0.9696 & 0.8129 & 2.2942 & 1.5019 & 1.1735 \\
\hline$k_{5}$ & 0.9685 & 0.7344 & 0.6712 & 1.3379 & 0.9714 & 0.8085 & 2.3356 & 1.5334 & 1.1979 \\
\hline$k_{6}$ & 0.9688 & 0.7583 & 0.6912 & 1.2818 & 0.9809 & 0.8331 & 2.1056 & 1.4233 & 1.1560 \\
\hline$k_{7}$ & 1.0045 & 0.7861 & 0.7078 & 1.3853 & 1.0441 & 0.8613 & 2.2242 & 1.5346 & 1.2311 \\
\hline$k_{8}$ & 0.8891 & 0.7134 & 0.6655 & 1.0748 & 0.8401 & 0.7774 & 1.9088 & 1.2657 & 1.0335 \\
\hline MLE & 1.6172 & 1.0400 & 0.9183 & 2.3961 & 1.5279 & 1.1320 & 4.5867 & 3.0125 & 2.1434 \\
\hline
\end{tabular}

Table 3. The AMSE values for different $k$ with optimal $d$ for $p=8$

\begin{tabular}{|c|c|c|c|c|c|c|c|c|c|}
\hline \multirow{2}{*}{$\begin{array}{l}\rho \\
n\end{array}$} & \multicolumn{3}{|c|}{0.9} & \multicolumn{3}{|c|}{0.95} & \multicolumn{3}{|c|}{0.99} \\
\hline & 50 & 100 & 200 & 50 & 100 & 200 & 50 & 100 & 200 \\
\hline$k_{1}$ & 3.6513 & 1.3095 & 0.6274 & 9.2806 & 1.8112 & 0.9489 & 20.6034 & 9.796 & 2.5225 \\
\hline$k_{2}$ & 3.6891 & 1.3368 & 0.6433 & 9.3837 & 1.8477 & 0.9729 & 20.8967 & 9.9669 & 2.584 \\
\hline$k_{3}$ & 4.8402 & 2.0404 & 0.9534 & 12.8513 & 2.8887 & 1.5463 & 32.2306 & 15.7924 & 4.8666 \\
\hline$k_{4}$ & 4.0841 & 1.5948 & 0.7691 & 10.6056 & 2.2816 & 1.1993 & 24.3956 & 11.7169 & 3.3359 \\
\hline$k_{5}$ & 3.9290 & 1.5937 & 0.7289 & 10.0599 & 2.1088 & 1.1405 & 21.4002 & 11.2511 & 3.1836 \\
\hline$k_{6}$ & 3.7811 & 1.6056 & 0.8181 & 9.0515 & 2.0722 & 1.2374 & 18.5028 & 9.2001 & 2.788 \\
\hline$k_{7}$ & 4.1232 & 1.9067 & 0.9507 & 9.7368 & 2.5571 & 1.4772 & 18.6701 & 10.38 & 3.6014 \\
\hline$k_{8}$ & 3.2690 & 1.1391 & 0.6451 & 8.1544 & 1.5275 & 0.8871 & 17.7195 & 8.0577 & 1.9944 \\
\hline MLE & 10.6802 & 4.1897 & 1.6451 & 30.2246 & 6.2970 & 2.7891 & 75.5528 & 35.5189 & 10.9108 \\
\hline
\end{tabular}




\section{LIU-TYPE LOGISTIC ESTIMATORS}

Table 4. The MAE values for different $k$ with optimal $d$ for $p=8$

\begin{tabular}{|c|c|c|c|c|c|c|c|c|c|}
\hline \multirow{2}{*}{$\frac{\rho}{n}$} & \multicolumn{3}{|c|}{0.9} & \multicolumn{3}{|c|}{0.95} & \multicolumn{3}{|c|}{0.99} \\
\hline & 50 & 100 & 200 & 50 & 100 & 200 & 50 & 100 & 200 \\
\hline$k_{1}$ & 1.2863 & 1.0359 & 0.7590 & 2.0771 & 1.1701 & 0.9270 & 3.1881 & 2.3832 & 1.3794 \\
\hline$k_{2}$ & 1.2997 & 1.0486 & 0.7693 & 2.1008 & 1.1847 & 0.9396 & 3.2277 & 2.4142 & 1.3991 \\
\hline$k_{3}$ & 1.7004 & 1.3507 & 0.9518 & 2.8578 & 1.5745 & 1.2091 & 4.7419 & 3.4694 & 2.066 \\
\hline$k_{4}$ & 1.4414 & 1.1654 & 0.8462 & 2.3822 & 1.3516 & 1.0517 & 3.7236 & 2.759 & 1.6368 \\
\hline$k_{5}$ & 1.4218 & 1.1648 & 0.8202 & 2.3309 & 1.2945 & 1.0236 & 3.4021 & 2.7069 & 1.6007 \\
\hline$k_{6}$ & 1.4011 & 1.1833 & 0.8773 & 2.1406 & 1.3002 & 1.0766 & 3.0293 & 2.3767 & 1.5059 \\
\hline$k_{7}$ & 1.5140 & 1.3062 & 0.9517 & 2.3067 & 1.4789 & 1.1836 & 3.0591 & 2.6345 & 1.7617 \\
\hline$k_{8}$ & 1.2016 & 0.9769 & 0.7801 & 1.8728 & 1.0800 & 0.9094 & 2.8762 & 2.0945 & 1.2177 \\
\hline MLE & 2.6659 & 1.9465 & 1.2458 & 4.5869 & 2.3624 & 1.6143 & 7.4523 & 5.4173 & 3.1146 \\
\hline
\end{tabular}

Table 5. The AMSE values for different $k$ with optimal $d$ for $p=12$

\begin{tabular}{|c|c|c|c|c|c|c|c|c|c|}
\hline \multirow{2}{*}{$\begin{array}{l}\rho \\
n\end{array}$} & \multicolumn{3}{|c|}{0.9} & \multicolumn{3}{|c|}{0.95} & \multicolumn{3}{|c|}{0.99} \\
\hline & 50 & 100 & 200 & 50 & 100 & 200 & 50 & 100 & 200 \\
\hline$k_{1}$ & 139.2714 & 4.5505 & 0.8339 & 379.1795 & 18.6147 & 1.1519 & 693.7565 & 29.1359 & 5.4585 \\
\hline$k_{2}$ & 139.5613 & 4.5923 & 0.8505 & 379.7215 & 18.7365 & 1.1746 & 695.7493 & 29.472 & 5.5575 \\
\hline$k_{3}$ & 148.6546 & 6.4653 & 1.5089 & 400.7071 & 24.0658 & 2.3208 & 768.2535 & 48.479 & 11.1368 \\
\hline$k_{4}$ & 144.0903 & 5.4530 & 1.1198 & 389.2163 & 20.9824 & 1.6189 & 723.2426 & 35.3703 & 7.631 \\
\hline$k_{5}$ & 134.0508 & 4.9199 & 1.0217 & 367.5066 & 19.4268 & 1.5013 & 662.6167 & 32.3825 & 6.7584 \\
\hline$k_{6}$ & 131.8453 & 4.8675 & 1.1637 & 362.3602 & 17.9196 & 1.6392 & 642.9656 & 26.1222 & 5.7143 \\
\hline$k_{7}$ & 134.5658 & 5.8933 & 1.4827 & 364.9559 & 20.5335 & 2.1233 & 643.2291 & 27.9867 & 7.7012 \\
\hline$k_{8}$ & 129.5472 & 3.9218 & 0.8216 & 359.3362 & 16.0274 & 1.0576 & 639.7481 & 23.8502 & 4.1557 \\
\hline MLE & 351.1918 & 11.6188 & 2.6647 & 935.6592 & 43.8108 & 4.1312 & 2048.4312 & 98.5518 & 22.1795 \\
\hline
\end{tabular}

Table 6. The MAE values for different $k$ with optimal $d$ for $p=12$

\begin{tabular}{|c|c|c|c|c|c|c|c|c|c|}
\hline \multirow{2}{*}{$\frac{\rho}{n}$} & \multicolumn{3}{|c|}{0.9} & \multicolumn{3}{|c|}{0.95} & \multicolumn{3}{|c|}{0.99} \\
\hline & 50 & 100 & 200 & 50 & 100 & 200 & 50 & 100 & 200 \\
\hline$k_{1}$ & 8.4860 & 1.5200 & 0.8701 & 12.8861 & 2.8799 & 1.0276 & 17.5892 & 3.7843 & 1.9859 \\
\hline$k_{2}$ & 8.5038 & 1.5332 & 0.8794 & 12.9122 & 2.9003 & 1.0382 & 17.6425 & 3.8241 & 2.0081 \\
\hline$k_{3}$ & 9.1525 & 2.0939 & 1.1998 & 13.9870 & 3.8298 & 1.4903 & 19.9062 & 5.9098 & 3.1408 \\
\hline$k_{4}$ & 8.8189 & 1.7987 & 1.0191 & 13.4347 & 3.2974 & 1.2276 & 18.4166 & 4.5498 & 2.4684 \\
\hline$k_{5}$ & 8.3426 & 1.6721 & 0.9695 & 12.6869 & 3.1359 & 1.1809 & 17.4483 & 4.3274 & 2.3113 \\
\hline$k_{6}$ & 8.2283 & 1.6989 & 1.0465 & 12.4874 & 2.9680 & 1.2456 & 16.7388 & 3.6399 & 2.1214 \\
\hline$k_{7}$ & 8.4317 & 1.9781 & 1.1911 & 12.6229 & 3.3975 & 1.4279 & 16.7299 & 3.9188 & 2.5582 \\
\hline$k_{8}$ & 8.0607 & 1.3933 & 0.8786 & 12.2928 & 2.5904 & 0.9990 & 16.5748 & 3.2482 & 1.7039 \\
\hline MLE & 13.9272 & 2.8855 & 1.5911 & 21.3777 & 5.3458 & 1.9804 & 33.1953 & 8.6407 & 4.4523 \\
\hline
\end{tabular}




\section{YASIN ASAR}

\section{Application}

An empirical application is demonstrated by using a data set taken from the web site of Statistics Sweden ${ }^{1}$. There are 290 municipalities in Sweden; eighty-three municipalities are considered. Those considered are the urban regions defined as the municipalities belonging to the Functional analysis regions Stockholm, Göteborg and Malmö, corresponding to the year 2012. The explanatory variables are defined as follows: $X_{1}$ is the population, $X_{2}$ is the number of unemployed people, $X_{3}$ is the number of newly constructed buildings, and $X_{4}$ is the number of bankrupt firms. A binary logistic regression model is set by using the dependent variable defined as follows: If there is an increase in the population of a municipality it is coded as 1 ; otherwise it is coded as 0 .

It is observed from Table 7 that the bivariate correlations among the regressors are high (all greater than 0.91) and the condition number of the data given by $\kappa=\sqrt{\lambda_{\max } / \lambda_{\text {min }}}=38.3274$ shows that there is severe multicollinearity problem with this data.

For different values of $k$ defined above and for the optimal shrinkage parameter $d_{\mathrm{opt}}$, the MSEs of the estimators are given in Table 8. It can be seen from that table that $k_{8}$ has the best performance in the sense of MSE reduction.

Also, $k_{5}$ and $k_{6}$ perform quite well. If the coefficients, standard errors of the estimators, and the corresponding t-values given in Table 9 are considered, it is seen that $k_{5}$ and $k_{6}$ have very low standard errors when compared to the other estimators. Moreover, t-values corresponding to $k_{5}$ and $k_{6}$ are larger than the others in absolute value which further shows the superiority of $k_{5}$ and $k_{6}$. Thus, $k_{5}$ and $k_{6}$ seem to be more practical for this data set. Finally, a graph of the MSE function versus $k$ is provided in Figure 1. According to Figure 1, the MSE of $\hat{\boldsymbol{\beta}}\left(k, d_{\mathrm{opt}}\right)$ has a decreasing tendency for the increasing values of the parameter $k$.

Table 7. The correlation matrix of the data used in the application

\begin{tabular}{rrrrr} 
& $\boldsymbol{X}_{\mathbf{1}}$ & $\boldsymbol{X}_{\mathbf{2}}$ & $\boldsymbol{X}_{\mathbf{3}}$ & $\boldsymbol{X}_{\mathbf{4}}$ \\
\hline $\boldsymbol{X}_{\mathbf{1}}$ & 1.0000 & 0.9937 & 0.9707 & 0.9514 \\
$\boldsymbol{X}_{\mathbf{2}}$ & 0.9937 & 1.0000 & 0.9527 & 0.9222 \\
$\boldsymbol{X}_{\mathbf{3}}$ & 0.9707 & 0.9527 & 1.0000 & 0.9765 \\
$\boldsymbol{X}_{\mathbf{4}}$ & 0.9514 & 0.9222 & 0.9765 & 1.0000 \\
\hline
\end{tabular}




\section{LIU-TYPE LOGISTIC ESTIMATORS}

Table 8. The MSEs of the estimators used in the application

\begin{tabular}{rrrrrrrrrr} 
& $\boldsymbol{k}_{\mathbf{1}}$ & $\boldsymbol{k}_{\mathbf{2}}$ & $\boldsymbol{k}_{\mathbf{3}}$ & $\boldsymbol{k}_{\mathbf{4}}$ & $\boldsymbol{k}_{\mathbf{5}}$ & $\boldsymbol{k}_{\mathbf{6}}$ & $\boldsymbol{k}_{\mathbf{7}}$ & $\boldsymbol{k}_{\mathbf{8}}$ & MLE \\
\hline MSE & 766.7641 & 776.6589 & 850.1244 & 813.5330 & 724.3047 & 693.2426 & 732.2801 & 687.6025 & 1894.307 \\
\hline
\end{tabular}

Table 9. Coefficients, standard errors, and t-values of the data

\begin{tabular}{rrrrrrrrrr}
\multicolumn{10}{c}{ Coefficients } \\
& $\boldsymbol{k}_{\mathbf{1}}$ & $\boldsymbol{k}_{\mathbf{2}}$ & $\boldsymbol{k}_{\mathbf{3}}$ & $\boldsymbol{k}_{\mathbf{4}}$ & $\boldsymbol{k}_{\mathbf{5}}$ & $\boldsymbol{k}_{\mathbf{6}}$ & $\boldsymbol{k}_{\mathbf{7}}$ & $\boldsymbol{k}_{\mathbf{8}}$ & $\mathbf{M L E}$ \\
\hline $\boldsymbol{\beta}_{1}$ & 9.5174 & 9.5364 & 9.7763 & 9.6347 & 9.4529 & 9.3549 & 9.4654 & 9.1923 & 25.3187 \\
$\boldsymbol{\beta}_{2}$ & -5.1346 & -5.1261 & -5.3010 & -5.1732 & -5.3543 & -5.9703 & -5.2813 & -6.3168 & -17.4101 \\
$\boldsymbol{\beta}_{3}$ & 2.3166 & 2.4417 & 3.3105 & 2.8941 & 1.7992 & 1.5479 & 1.8892 & 1.4059 & 3.8671 \\
$\boldsymbol{\beta}_{4}$ & -5.7236 & -5.8749 & -6.8082 & -6.3768 & -4.9382 & -4.0628 & -5.1086 & -3.9790 & -10.9671 \\
\hline
\end{tabular}

\begin{tabular}{rrrrrrrrrr}
\multicolumn{8}{c}{ Standard errors } \\
& $\boldsymbol{k}_{\mathbf{1}}$ & $\boldsymbol{k}_{\mathbf{2}}$ & $\boldsymbol{k}_{\mathbf{3}}$ & $\boldsymbol{k}_{\mathbf{4}}$ & $\boldsymbol{k}_{\mathbf{5}}$ & $\boldsymbol{k}_{\mathbf{6}}$ & $\boldsymbol{k}_{\mathbf{7}}$ & $\boldsymbol{k}_{\mathbf{8}}$ & MLE \\
\hline $\boldsymbol{\beta}_{1}$ & 1.2685 & 1.2749 & 1.3382 & 1.3035 & 1.2496 & 1.2513 & 1.2519 & 1.2550 & 3.4576 \\
$\boldsymbol{\beta}_{2}$ & 1.0505 & 1.0579 & 1.1134 & 1.0852 & 1.0167 & 0.9884 & 1.0235 & 0.9840 & 2.7109 \\
$\boldsymbol{\beta}_{3}$ & 0.8606 & 0.9019 & 1.1718 & 1.0450 & 0.6694 & 0.5164 & 0.7069 & 0.4866 & 1.3404 \\
$\boldsymbol{\beta}_{4}$ & 0.7752 & 0.8023 & 0.9745 & 0.8942 & 0.6404 & 0.5067 & 0.6686 & 0.4764 & 1.3124 \\
\hline
\end{tabular}

\begin{tabular}{|c|c|c|c|c|c|c|c|c|c|}
\hline \multicolumn{10}{|c|}{ t-values } \\
\hline & k1 & k2 & k3 & k4 & k5 & k6 & k7 & k8 & MLE \\
\hline $\boldsymbol{\beta}_{1}$ & 7.5029 & 7.4804 & 7.3054 & 7.3911 & 7.5650 & 7.4761 & 7.5609 & 7.3244 & 7.3226 \\
\hline $\boldsymbol{\beta}_{2}$ & -4.8877 & -4.8458 & -4.7611 & -4.767 & -5.2665 & -6.0405 & -5.1599 & -6.4195 & -6.4222 \\
\hline $\boldsymbol{\beta}_{3}$ & 2.6919 & 2.7073 & 2.8252 & 2.7694 & 2.6878 & 2.9977 & 2.6726 & 2.8891 & 2.8850 \\
\hline $\boldsymbol{\beta}_{4}$ & -7.3835 & -7.3224 & -6.9862 & -7.1315 & -7.7113 & -8.0179 & -7.6402 & -8.3516 & -8.3567 \\
\hline
\end{tabular}

\section{Summary and Conclusion}

The benefits of Liu-type estimators in logistic regression were shown in the case of multicollinearity. The optimal shrinkage parameter $d_{\text {opt }}$ used in the Liu-type estimator was defined in Huang (2012). The Monte Carlo experiment was used to evaluate the early proposed ridge regression parameters in logistic regression. Results show that the estimators chosen from the literature outperform MLE for all of the cases taken into consideration when the optimal shrinkage parameter $d_{\text {opt }}$ is used. AMSE and MAE values of MLE become inflated when the correlation increases and the sample size decreases. Thus, researchers are advised to use Liutype logistic estimators with the optimal shrinkage parameter in place of MLE in the presence of multicollinearity. The best performance from the estimator will be obtained if $k_{8}$ is used. 
YASIN ASAR

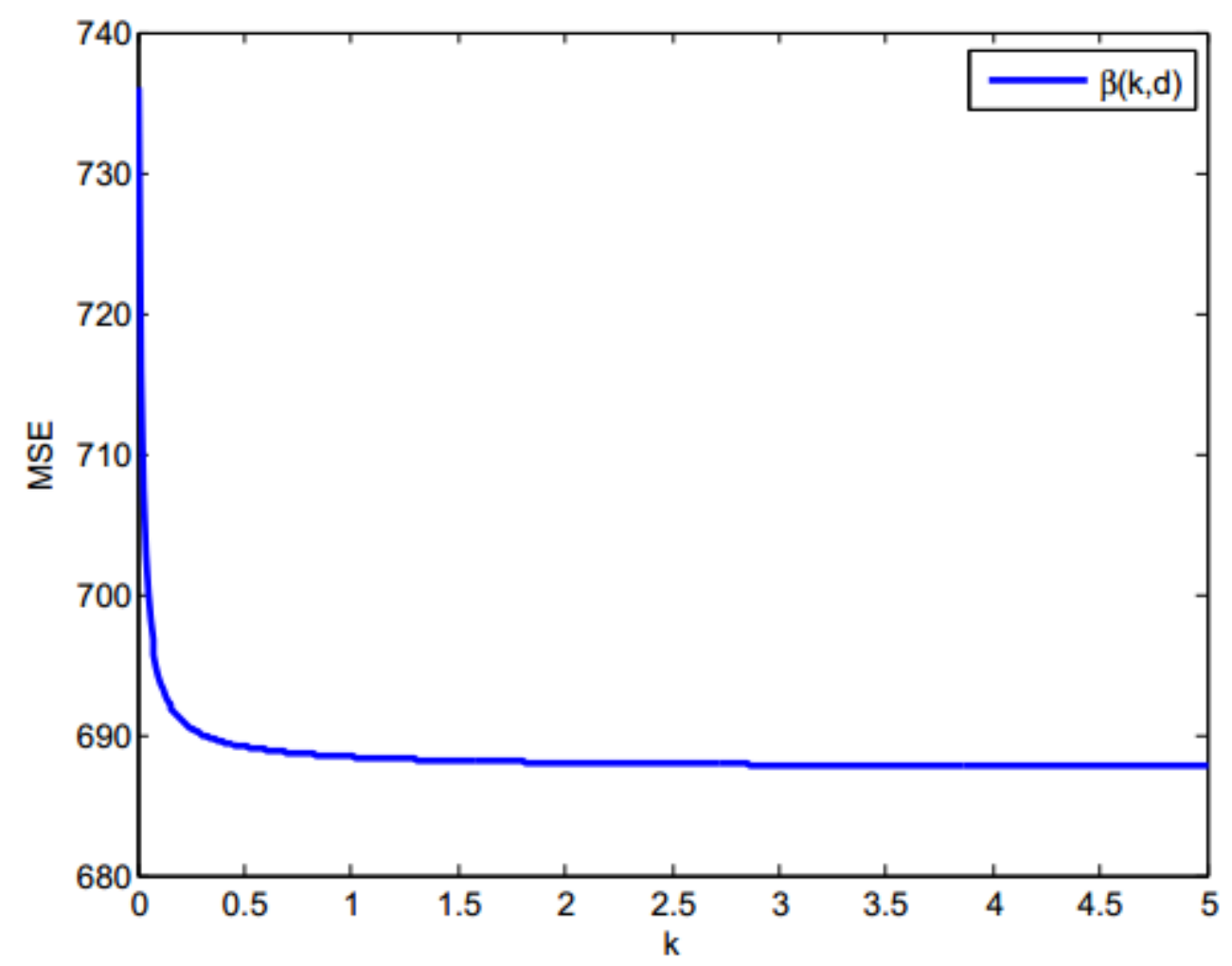

Figure 1. The MSE of $\hat{\boldsymbol{\beta}}(k, d)$ function versus $k$

\section{Footnotes}

1. The data used in this article may be accessed through the following website: http://www.scb.se/sv_/Hitta-statistik/

\section{References}

Alkhamisi, M., Khalaf, G., \& Shukur, G. (2006). Some modifications for choosing ridge parameters. Communications in Statistics-Theory and Methods, 35(11), 2005-2020. doi: 10.1080/03610920600762905

Alkhamisi, M., \& Shukur, G. (2008). Developing ridge parameters for SUR model. Communications in Statistics-Theory and Methods, 37(4), 544-564. doi: 10.1080/03610920701469152 


\section{LIU-TYPE LOGISTIC ESTIMATORS}

Asar, Y., Karaibrahimoğlu, A., \& Genç, A. (2014). Modified ridge regression parameters: A comparative Monte Carlo study. Hacettepe Journal of Mathematics and Statistics, 43(5), 827-841. Retrieved from http://www.hjms.hacettepe.edu.tr/uploads/7742744a-b036-456d-83671a3eab73e172.pdf

Hoerl, A. E., \& Kennard, R. W. (1970). Ridge regression: Biased estimation for nonorthogonal problems. Technometrics, 12(1), 55-67. doi:

10.1080/00401706.1970.10488634

Huang, J. (2012). A simulation research on a biased estimator in logistic regression model. In Z. Li, X. Li, Y. Liu, \& Z. Cai (Eds.), Computational Intelligence and Intelligent Systems (389-395). Berlin, Germany: Springer. doi: 10.1007/978-3-642-34289-9_43

Inan, D., \& Erdogan, B. E. (2013). Liu-type logistic estimator. Communications in Statistics-Simulation and Computation, 42(7), 1578-1586. doi: 10.1080/03610918.2012.667480

Kibria, B. M. G. (2003). Performance of some new ridge regression estimators. Communications in Statistics-Simulation and Computation, 32(2), 419-435. doi: 10.1081/SAC-120017499

Kibria, B. M. G., Månsson, K., \& Shukur, G. (2012). Performance of some logistic ridge regression estimators. Computational Economics, 40(4), 401-414. doi: 10.1007/s10614-011-9275-X

Liu, K. (1993). A new class of biased estimate in linear regression. Communications in Statistics-Theory and Methods, 22(2), 393-402. doi: 10.1080/03610929308831027

Liu, K. (2003). Using Liu-type estimator to combat collinearity. Communications in Statistics-Theory and Methods, 32(5), 1009-1020. doi: 10.1081/STA-120019959

Månsson, K., Kibria, B. M. G., \& Shukur, G. (2012). On Liu estimators for the logit regression model. Economic Modelling, 29(4), 1483-1488. doi: 10.1016/j.econmod.2011.11.015

Månsson, K., \& Shukur, G. (2011). On ridge parameters in logistic regression. Communications in Statistics-Theory and Methods, 40(18), 33663381. doi: 10.1080/03610926.2010.500111

Muniz, G., \& Kibria, B. M. G. (2009). On some ridge regression estimators: An empirical comparisons. Communications in Statistics-Simulation and Computation, 38(3), 621-630. doi: 10.1080/03610910802592838 


\section{YASIN ASAR}

Newhouse, J. P., \& Oman, S. D. (1971). An evaluation of ridge estimators: A report prepared for the United States Air Force Project Rand (P-716-PR).

Retrieved from

http://www.rand.org/content/dam/rand/pubs/reports/2007/R716.pdf

Schaefer, R. L., Roi, L. D., \& Wolfe, R. A. (1984). A ridge logistic

estimator. Communications in Statistics-Theory and Methods, 13(1), 99-113. doi: 10.1080/03610928408828664 